\title{
Can a Second Order Bandpass Sigma Delta Modulator Achieve High Signal-to-noise Ratio for Lowpass Inputs?
}

\author{
Charlotte Yuk-Fan Ho \\ Telephone: +44 (0)20 78827986 Fax: +44 (0)20 78827997 Email: charlotte.ho@elec.qmul.ac.uk \\ Department of Electronic Engineering, Queen Mary, University of London, Mile End Road, London, E1 4NS, United Kingdom. \\ *Bingo Wing-Kuen Ling \\ Telephone: +44 (0)20 78482294 Fax: +44 (0)20 78482932 Email: wing-kuen.ling@kcl.ac.uk
} Department of Electronic Engineering, Division of Engineering, King's College London, Strand, London, WC2R 2LS, United Kingdom.

\begin{abstract}
In this letter, it is found that a second order interpolative bandpass sigma delta modulator (SDM) can achieve high signal-to-noise ratio (SNR) even if input signals have lowpass characteristics.
\end{abstract}

Keyword: Sigma delta modulator, signal-to-noise ratio, limit cycle

\section{Introduction}

Institutively, second order SDMs usually achieve lower SNR than high order ones because high order loop filters can achieve better noise shaping characteristics. Moreover, the signal transfer function should be designed to have large values and the noise transfer function should be designed to have small values at the passband of loop filters in order to achieve good noise shaping characteristics, so SNR should be high if input signal bands match passbands of loop filters and low otherwise. Based on this argument, one may expect that SNR will be low when input signals have lowpass characteristics while loop filters have bandpass characteristics. 
However, since the above argument is based on the noise shaping theory which is formulated using a linear model, while quantizers in SDMs are nonlinear components, the linear model may not explain nonlinear system behaviors. In this letter, a counterexample is given to illustrate that a second order bandpass interpolative SDM may also give a very high SNR for lowpass inputs.

\section{Main results}

Consider the following second order interpolative SDM:

$$
F(z)=\frac{10^{7}\left(0.1118520172881933 z^{-1}-1.234413507514501 z^{-2}\right)}{1-0.999999975380456 z^{-1}+0.9999999873131996 z^{-2}}
$$

Figure 1 shows the magnitude response of the loop filter. It can be seen from the figure that the loop filter has a bandpass characteristics with peak frequency located round $\frac{\pi}{3}$. We assume that the oversampling ratio of the SDM is 64 because it is the most common value used in the audio industry [1]. Also, we use the delays of the output of the loop filter as state variables, that is, $\mathbf{x}(k) \equiv[y(k-2) \quad y(k-1)]^{T}$, in which $\mathbf{x}(k)$ and $y(k)$ are the state vectors and the output of the loop filter, respectively. We assume that the SDM is initially at rest, so $\mathbf{x}(0)=\mathbf{0}$. The reason for choosing the delays of the output of the loop filter as state variables and assume initially at rest is because zero output at the loop filter is the most common situation in a real case. Figure 2 shows the SNR performance of the SDM. Here, we adopt the definition of SNR defined in [2], that is, the inputs are sinusoidal signals with frequency $\frac{\pi}{96}$, which are regarded as lowpass inputs compared to the peak frequency of the loop filter. It can be seen from Figure 2 that this SDM can achieve SNR as high as $323.5228 \mathrm{~dB}$ for input magnitude less or equal to 1.02 , while $323.5037 \mathrm{~dB}$ for input magnitude larger than 1.02. Compared this result to a fifth order SDM [1], it can only 
achieve an average of $111.1987 \mathrm{~dB}$ for an input magnitude less than or equal to 0.59 , and $-41.6297 \mathrm{~dB}$ for above. To compare the result with the second order SDM with two DC poles and a zero located at 0.5 , it can only achieve an average of $65.6404 \mathrm{~dB}$ for an input magnitude less than or equal to 1.08 , and $8.5519 \mathrm{~dB}$ for above. Hence, this second order SDM produces better performance than a fifth order SDM [1] and the two DC poles second order interpolative SDM.

The SDM can achieve such high SNR because of exhibiting the limit cycle behavior. If the quantizer output is periodic with the period being an integer multiple of the input signal, then the input of the loop filter is periodic with the period equal to that of the quantizer output. Hence, the quantizer input is also periodic with the same period as its output. In this case, the SNR of the SDM can be very high even though the loop filter is second order and has the bandpass characteristics. To illustrate this phenomenon, the magnitude spectra of the filter output, the quantizer output and the input signal are shown in Figure 3, in which the period of both the quantizer output and the input signal is found to be 192 .

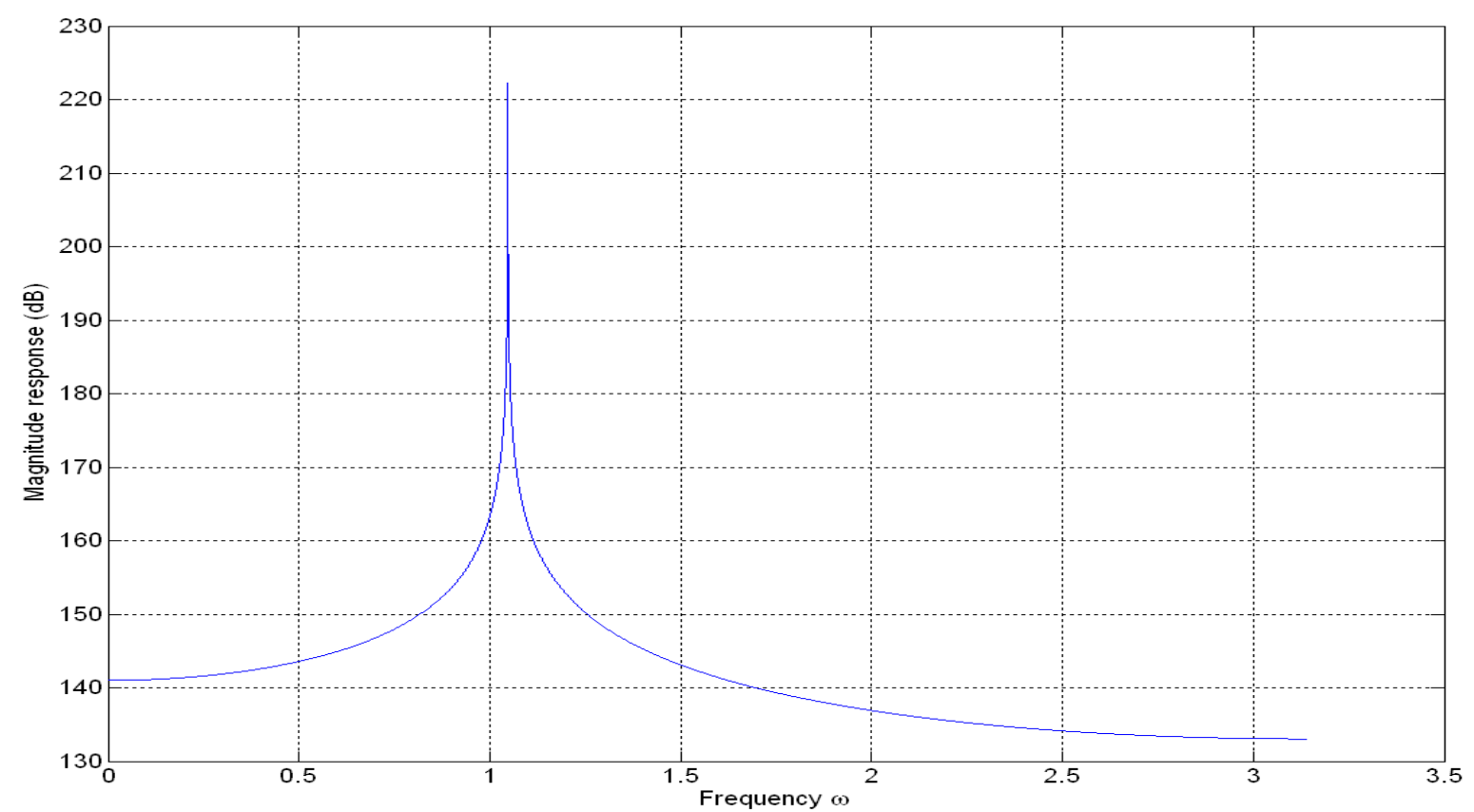

Figure 1: Magnitude response of the loop filter. 


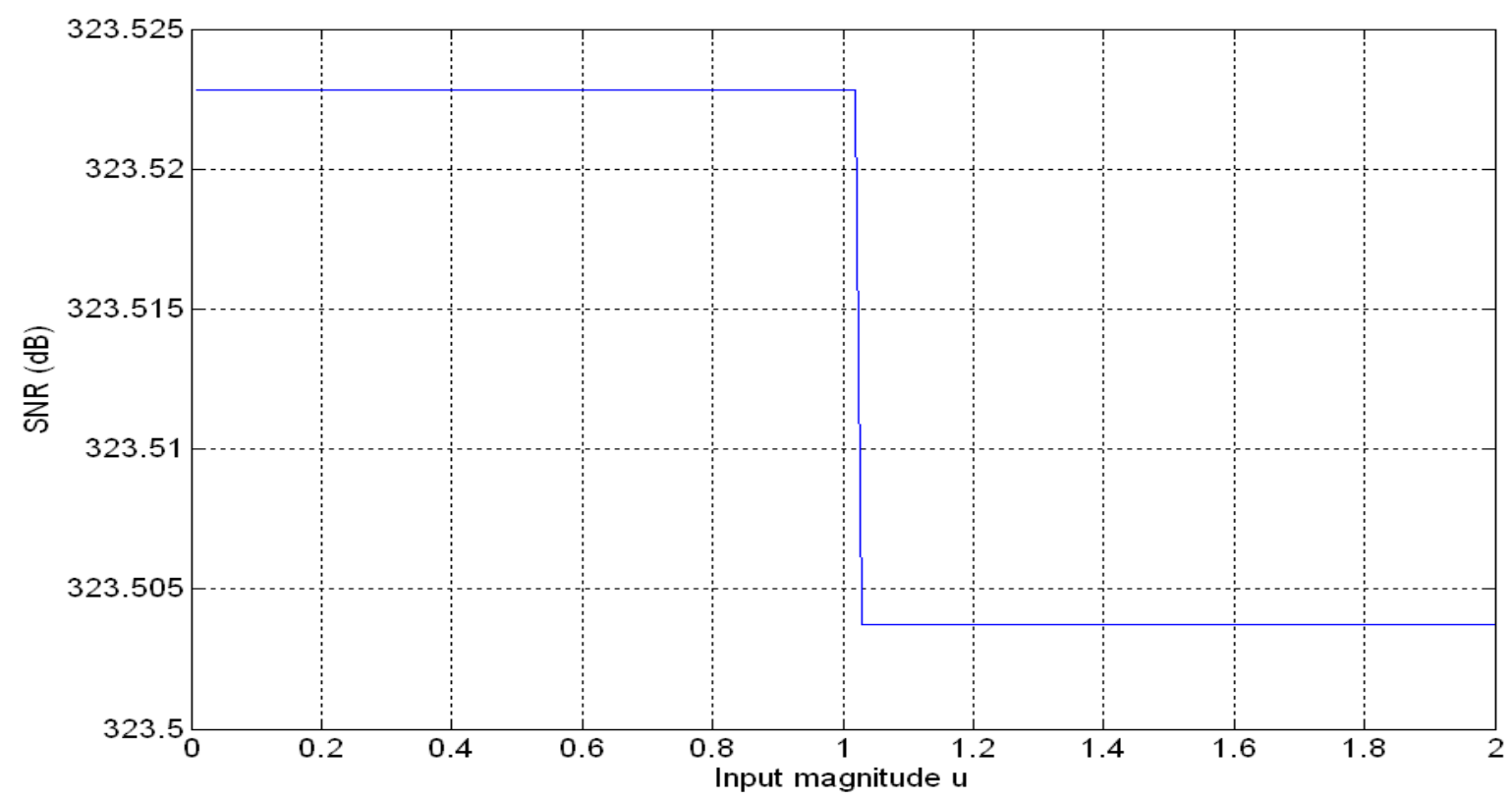

Figure 2: SNR of the second order interpolative SDM.
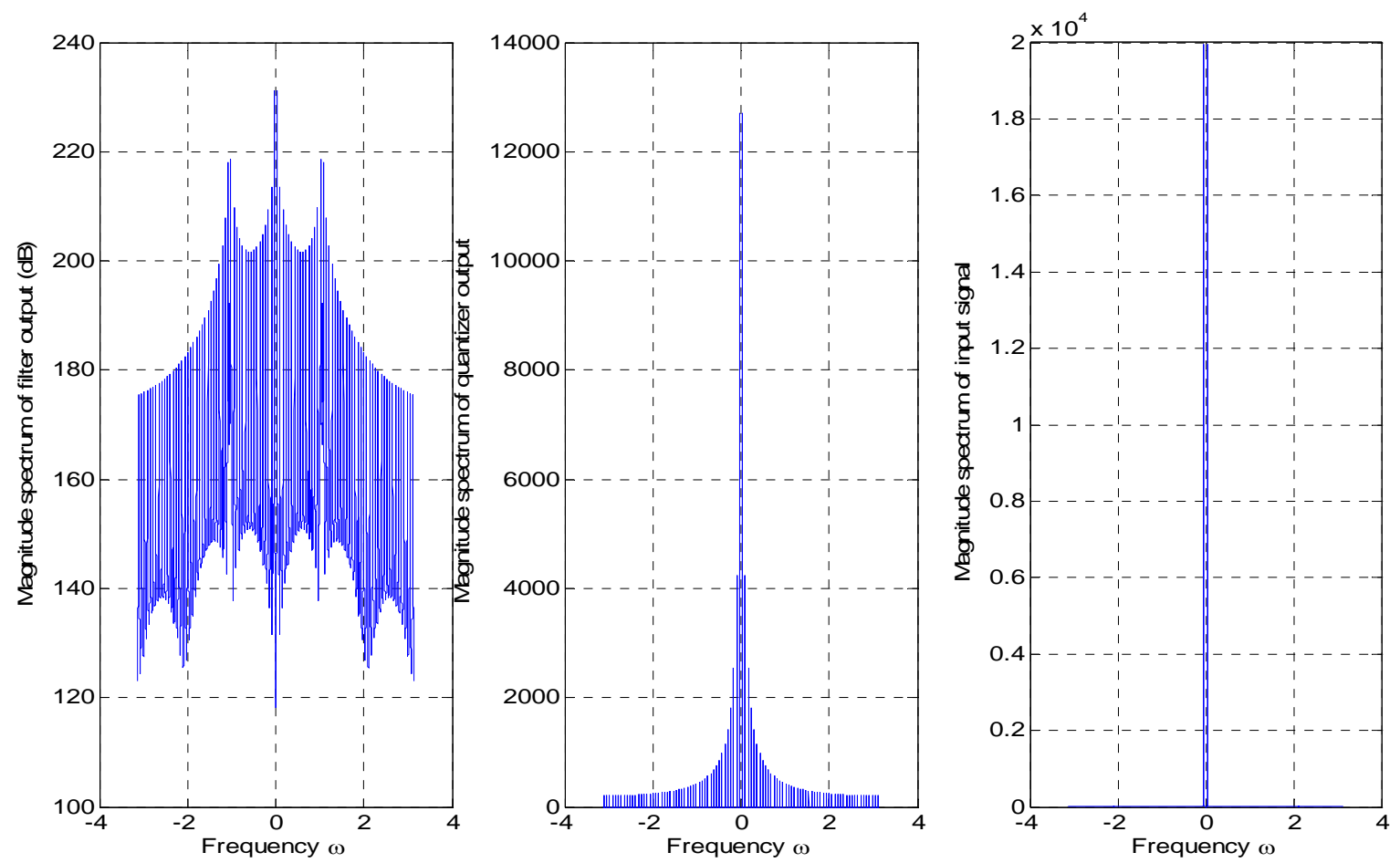

Figure 3: Magnitude spectra of the filter output, the quantizer output and the input signal of the second order interpolative SDM.

\section{Conclusion}

In this letter, a counterexample is given to illustrate that a second order bandpass interpolative SDM can achieve very high SNR even though input signals have 
lowpass characteristics.

\section{Acknowledgment}

The work obtained in this letter was supported by a research grant from Queen Mary, University of London.

\section{References}

[1] Derk Reefman and Erwin Janssen, "Signal processing for direct stream digital: a tutorial for digital sigma delta modulation and 1-bit digital audio processing,” Philips Research, Eindhoven, White Paper, 2002.

[2] Richard Schreier, The delta-sigma modulators toolbox version 6.0, Analog Devices Inc., $1^{\text {st }}$ Jan 2003. 